\title{
REVIEW
}

\section{4-3-3 and its binding partners are regulators of protein-protein interactions during spermatogenesis}

\author{
Shengyi Sun, Elissa W P Wong, Michelle W M Li, Will M Lee ${ }^{\mathbf{1}}$ and C Yan Cheng \\ The Mary M Wohlford Laboratory for Male Contraceptive Research, Center for Biomedical Research, Population Council, 1230 York Avenue, New York, \\ New York 10065, USA \\ ${ }^{1}$ School of Biological Sciences, University of Hong Kong, Hong Kong, People's Republic of China \\ (Correspondence should be addressed to C Y Cheng; Email: y-cheng@popcbr.rockefeller.edu)
}

\begin{abstract}
During spermatogenesis, spermiation takes place at the adluminal edge of the seminiferous epithelium at stage VIII of the epithelial cycle during which fully developed spermatids (i.e. spermatozoa) detach from the epithelium in adult rat testes. This event coincides with the migration of preleptotene/leptotene spermatocytes across the blood-testis barrier from the basal to the apical (or adluminal) compartment. At stage XIV of the epithelial cycle, Pachytene spermatocytes (diploid, 2n) differentiate into diplotene spermatocytes (tetraploid, 4n) in the apical compartment of the epithelium, which begin meiosis I to be followed by meiosis II to form spermatids (haploid, 1n) at stage XIV of the epithelial cycle. These spermatids, in turn, undergo extensive morphological changes and traverse the seminiferous epithelium until they differentiate into elongated spermatids. Thus, there are extensive changes at the Sertoli-Sertoli and Sertoli-germ cell interface via protein 'coupling' and
\end{abstract}

'uncoupling' between cell adhesion protein complexes, as well as changes in interactions between integral membrane proteins and their peripheral adaptors, regulatory protein kinases and phosphatases, and the cytoskeletal proteins. These precisely coordinated protein-protein interactions affect cell adhesion and cell movement. In this review, we focus on the 14-3-3 protein family, whose members have different binding partners in the seminiferous epithelium. Recent studies have illustrated that 14-3-3 affects protein-protein interactions in the seminiferous epithelium, and regulates cell adhesion possibly via its effects on intracellular protein trafficking and cell-polarity proteins. This review provides a summary on the latest findings regarding the role of 14-3-3 family of proteins and their potential implications on spermatogenesis. We also highlight research areas that deserve attentions by investigators.

Journal of Endocrinology (2009) 202, 327-336

\section{Introduction}

During spermatogenesis, in addition to self-renewal of spermatogonia via i) mitosis, type $\mathrm{B}$ spermatogonia will differentiate into primary spermatocytes, which eventually enter ii) meiosis to give rise to haploid spermatids. Spermatids begin their maturation in a process known as iii) spermiogenesis behind the blood-testis barrier (BTB) that transforms round spermatids into elongated spermatids (from step 1 to step 19 spermatids in rats) with profound changes in the spermatid head (condensation of the chromatin materials and formation of the acrosome) and elongation of the tail until iv) spermiation. At spermiation, fully developed spermatids (i.e. spermatozoa) will leave the seminiferous epithelium, entering the tubule lumen to undergo maturation in the epididymis (for a review, see Hess \& Franca 2008). During these four distinctive phases, extensive restructuring occurs at the
Sertoli-Sertoli and Sertoli-germ cell interface across the entire seminiferous epithelium in both the basal and apical compartments as well as in the BTB in adult mammalian testes (for reviews, see Cheng \& Mruk 2002, Mruk \& Cheng 2004). Recent studies have shown that efficient restructuring at the cell-cell interface facilitates both the migration of developing spermatids in the seminiferous epithelium during spermiogenesis and spermiation, and the transit of primary preleptotene spermatocytes across the BTB. Such dynamics are mediated, at least in part, via changes in the proteinprotein interactions between integral membrane proteins and their adaptors (e.g. $\alpha_{-}, \beta-, \gamma$-catenins, and $\mathrm{ZO}-1$ that tether integral membrane proteins, such as $N$-cadherin, occludin, JAMs, and nectins, to the cytoskeletal proteins; catenins and ZO-1 also recruit signaling molecules, such as protein kinases and/or phosphatases, to the same site; for reviews, see Lee \& Cheng 2004, Mruk \& Cheng 2004, Zhang et al. 2005). 
The net results of these interactions determine whether the integral membrane proteins are structurally linked to the cytoskeletal network, thus affecting the status of cell adhesion in the epithelium. Consequently, it is of interest to identify and investigate proteins that are crucial to protein-protein interactions at the cell-cell interface in the seminiferous epithelium. In this minireview, we focus on a protein family named 14-3-3, which has recently been shown to be a crucial regulator of protein-protein interactions in various epithelial and endothelial cells in mammals. While many of the studies were done in organs other than the testes, except for a few reports (Wine \& Chapin 1999, Chapin et al. 2001, W P Wong \& C Y Cheng, unpublished observations), we thought it pertinent to critically evaluate recent data in the field to provide a framework with which functional studies can be performed to tackle the role of 14-3-3 in spermatogenesis.

\section{Background and physico-chemical properties of 14-3-3}

14-3-3 proteins comprise a family of small acidic proteins $(\sim 30 \mathrm{kDa})$ found in virtually all tissues of both invertebrates and vertebrates. They were discovered in 1967 during a systemic classification of brain proteins, and named by their specific location following diethyl aminoethyl (DEAE)-cellulose chromatography and starch gel electrophoresis (Moore \& Perez 1967). The 14-3-3 family members are highly conserved, with seven isoforms identified in mammals, at least 12 isoforms in plants, two isoforms in Drosophila, and two isoforms in yeast (Wang \& Shakes 1996, Rosenquist et al. 2000, 2001). In mammals, despite their highest expression in the central nervous system, 14-3-3 protein family exists ubiquitously in almost all other tissues, especially in the intestines and testis (Boston et al. 1982). Different 14-3-3 isoforms display a certain degree of tissue specificity, and they are present at different concentrations in tissues (Perego \& Berruti 1997).

The seven isoforms identified in mammals ( $\beta$-beta, $\gamma$-gamma, $\varepsilon$-epsilon, $\zeta$-zeta, $\eta$-eta, $\theta$-theta, and $\sigma$-sigma; Table 1) share about $50 \%$ amino acid identity and, consequently, highly similar protein conformations to form either homodimers or heterodimers that serve as the functional protein units (Jones et al. 1995, Chaudhri et al. 2003). Each 14-3-3 monomer is composed of nine $\alpha$-helixes and relatively unconserved carboxyl-terminal and aminoterminal regions (Aitken 2006). Theoretically, the rigid helical structure may provide the functional basis for target binding, whereas the flexible carboxyl terminal allows for alternative binding activities (Bridges \& Moorhead 2004). The amino-terminal region forms the interface for dimerization, thus determining the specific combinations of isoforms (Bridges \& Moorhead 2004, Aitken 2006).

Dimeric 14-3-3 proteins have a cup-shaped conformation, with two highly conserved amphipathic grooves as target-binding pockets (Liu et al. 1995, Xiao et al. 1995, Rittinger et al. 1999). From the peptide library, two highaffinity phosphorylation-dependent motifs were identified among a variety of 14-3-3 binding partners, which are RSXpSXP and RXXXpSXP (X, any amino acid; pS, phosphoserine, which can be substituted by phosphothreonine, pT; R, Arg, S, Ser and P, Pro; Yaffe et al. 1997, Rittinger et al. 1999, Bridges \& Moorhead 2004). Moreover, although constituting only a small proportion, a third phosphorylation-independent binding motif, which still binds to the same binding pocket on 14-3-3, has been reported (Petosa et al. 1998, Masters et al. 1999).

To date, more than 200 binding partners of 14-3-3 have been reported, most of which were identified based on proteomic studies. These 14-3-3 binding proteins are involved in a wide range of cellular activities, such as transcription, protein synthesis, metabolic pathways, cell cycle, cell signaling, cytoskeletal organization, and cellular trafficking (Dougherty \& Morrison 2004, Jin et al. 2004, Kjarland et al. 2006, Shikano et al. 2006). Notably, such 14-3-3 interactome studies were performed in different cell types, with different methodologies (such as co-immunoprecipitation or affinity chromatography), and with the interacting proteins targeting different 14-3-3 isoforms (Kjarland et al. 2006). Thus, upon comparison, only a small proportion of these parameters (e.g. binding partners of 14-3-3) overlap between different 14-3-3 isoforms (Bridges \& Moorhead 2005). However, in a study utilizing the same method but targeting different isoforms, a small degree of overlapping was still reported, which seems to suggest isoform specificity even though there is functional redundancy between different 14-3-3 isoforms (Benzinger et al. 2005, Bridges \& Moorhead 2005, Kjarland et al. 2006). This isoform specificity has also been substantiated in some functional studies, which are summarized in Table 1. These observations thus illustrate that $14-3-3$ is a crucial molecule in conferring protein-protein interactions in different cells. Since it is found abundantly in the testes (Table 1), it is not surprising that this protein may be crucial to Sertoli-Sertoli and/or Sertoli-germ cell interactions during spermatogenesis. In fact, recent studies have demonstrated the presence of several 14-3-3 members in the testis, such as $14-3-3 \theta, 14-3-3 \beta$, and $14-3-3 \zeta$; and 14-3-30 was found in both Sertoli and germ cells (W P Wong \& C Y Cheng, unpublished observations). In a study using immunohistochemistry and fluorescent microscopy (Fig. 1), it was shown that $14-3-3 \theta$ was localized prominently at the elongating spermatid-Sertoli cell interface, which is consistent with its localization at the apical ectoplasmic specialization (apical ES, a testis-specific atypical adherens junction, AJ, type (for review, see Wong et al. 2008b)). Meanwhile, some 14-3-30 staining was detected in the basal compartment, consistent with its presence at the BTB (Fig. 1; W P Wong \& C Y Cheng, unpublished observations). 
Table 1 Different isoforms of 14-3-3 and their physiological function

\section{Physico-chemical characteristics and functions}

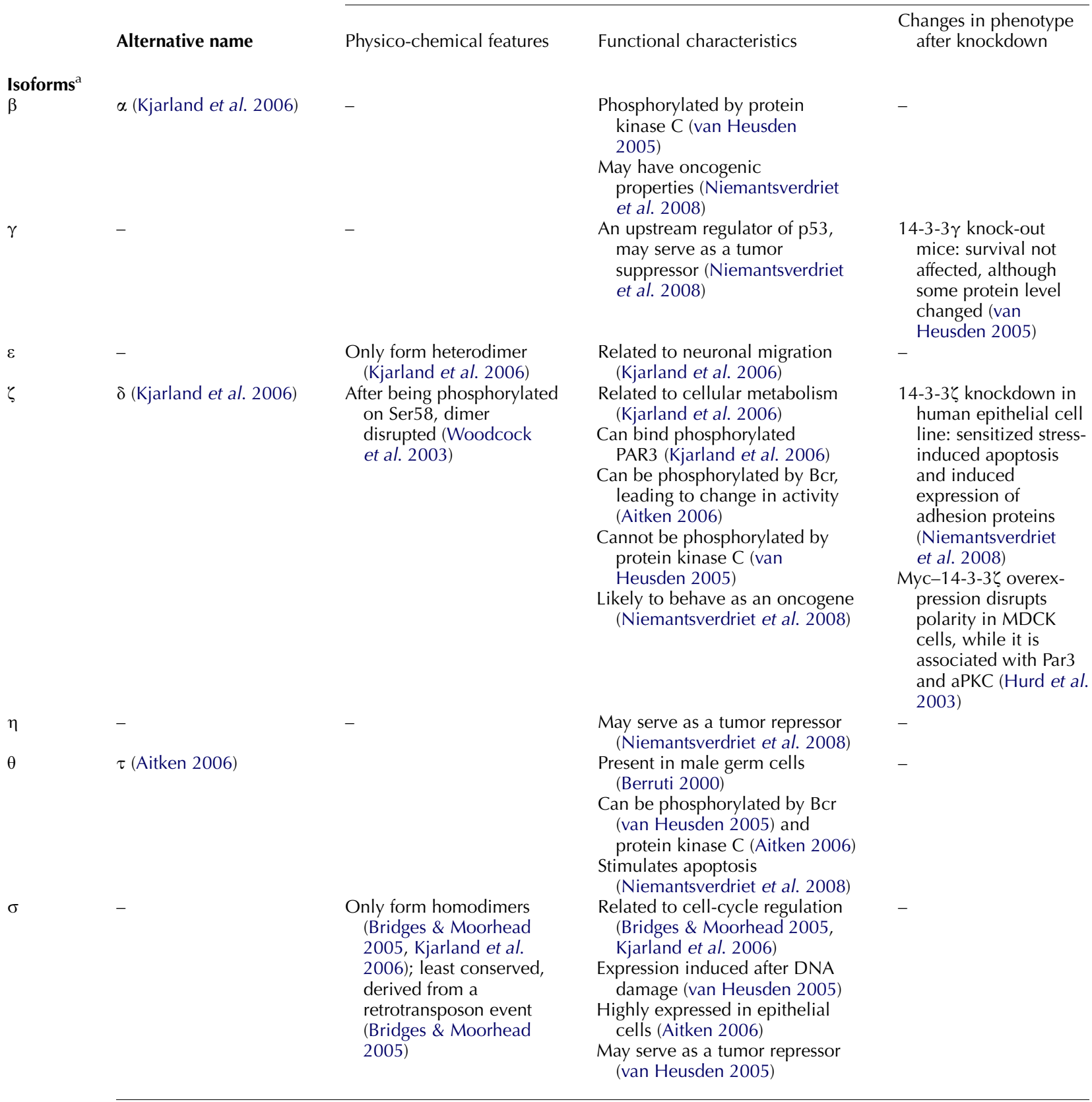

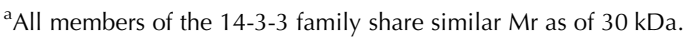

\section{Unique features of $14-3-3$}

The molecular mechanisms utilized by $14-3-3$ proteins to regulate cellular functions can be classified as clamping, masking, or scaffolding, all of which are mediated via protein-protein interactions between 14-3-3 and its target proteins. No clear evidence exists for any enzymatic activities of 14-3-3 (Fu et al. 2000, Bridges \& Moorhead 2004, Mrowiec \& Schwappach 2006, Shikano et al. 2006).

Clamping describes the process through which the conformation of the target protein is modified by the binding of 14-3-3. This action is facilitated by the rigid helical structure of 14-3-3, which shows little change after being bound with a ligand (Liu et al. 1995, Yaffe 2002). 

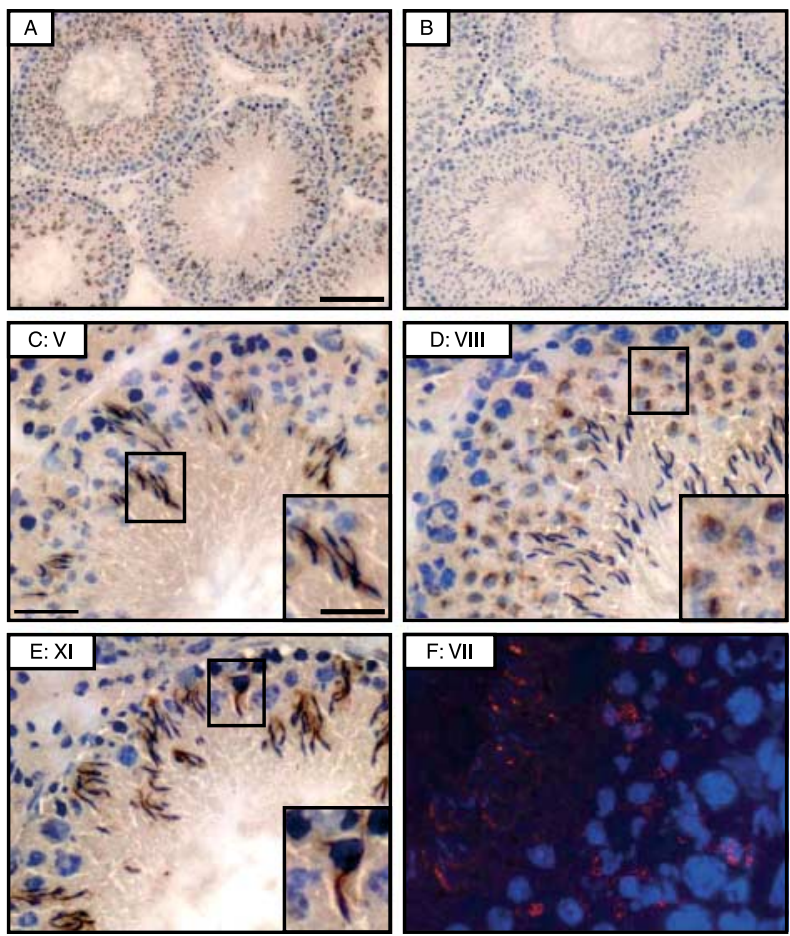

Figure 1 A study to assess the cellular localization of 14-3-3 in the seminiferous epithelium of adult rat testes. (A-E) Immunohistochemical localization of 14-3-30 in normal adult rat testes, in which frozen cross sections ( $\sim 7 \mu \mathrm{m}$ thick) were immunostained using an anti-14-3-30 IgG (Santa Cruz Biotechnology, Cat. Sc-732; Lot.

A2908) (A and C-E) or normal rabbit IgG (B). Low magnification of testes stained with anti-14-3-30 polyclonal antibody (A) or normal rabbit $\lg \mathrm{B}(\mathrm{B})$ illustrating the presence of $14-3-3 \theta$ in the seminiferous epithelium. In higher magnification, intense staining is obvious surrounding the heads of elongating spermatids at the apical ES site (C and E) as well as round spermatids (D) and the basal compartment consistent with its presence at the BTB. Bar in $(A)=100 \mu \mathrm{m}$, which applies to $(B)$, bar in $(C)=30 \mu \mathrm{m}$, which applies to $(D$ and $E)$, bar in inset in $(C)=15 \mu \mathrm{m}$, which applies to insets in (D and $\mathrm{E})$ and $(\mathrm{F})$. A study by immunofluorescence microscopy further illustrates the localization of 14-3-30 in seminiferous epithelium of normal adult rat testes, by using frozen testis cross sections ( $\sim 7 \mu \mathrm{m}$ thick) stained with anti-14-3-30 IgG. CY3-conjugated donkey anti-rabbit secondary antibody (red fluorescence) was used for visualizing 14-3-30, confirming the existence of 14-3-30 at the apical ES in the seminiferous epithelium in a stage VII tubule. Cell nuclei were stained by DAPI. Bar in $(F)=12 \mu \mathrm{m}$. The Roman numeral after C, D, E and F represents the seminiferous epithelial stage of the tubule.

Thus, 14-3-3 binding helps to reshape a target protein and, consequently, alters its properties. An example of such a clamping target is serotonin- $N$-acetyltransferase, which is stabilized and kept in an active conformation by 14-3-3 binding (Obsil et al. 2001). Notably, the binding between serotonin- $N$-acetyltransferase and $14-3-3$ is mediated by two phosphorylation sites, which bind to the two separate subunits of a 14-3-3 dimer (Ganguly et al. 2005). Along with the activation of enzymes, 14-3-3 clamping may also inactivate catalytic activities or alter the distal conformation of the target proteins (Moorhead et al. 1996, Athwal et al. 1998, McKinsey et al. 2001, Bridges \& Moorhead 2004).

The masking of a specific region in a target protein by 14-3-3 may alter a sequence-specific property of the target protein, such that access by other possible interacting proteins is blocked. This process can be viewed as a competition between 14-3-3 and any other interacting proteins for the target protein (Mackintosh 2004). For instance, 14-3-3 binding masks the nuclear localization signal of Cdc25C protein (a cell-cycle regulator) and sequesters the protein in the cytoplasm (Peng et al. 1997, Kumagai \& Dunphy 1999).

14-3-3 also employs a scaffolding mechanism by bringing two target proteins into proximity and co-localizing them at the same cellular site to carry out their cellular effects. This mechanism is plausibly based on the dimeric structure of 14-3-3, which has two independent binding pockets for target proteins. For instance, if a protein kinase and its substrate are bound in the two binding pockets on 14-3-3 simultaneously, the catalytic activity of the kinase is promoted, given the proximity of the enzyme and its substrate. Moreover, the catalytic activity may be tightly regulated owing to the restricted spatial orientation of the two 14-3-3 binding proteins (Bridges \& Moorhead 2004). On the other hand, regulation may be carried out to allow for scaffolding, because both the enzyme and the substrate need to be partially phosphorylated before they bind to 14-3-3 (Bridges \& Moorhead 2004). Scaffolding is possibly an important function for cellular signaling processes, which involve various regulated kinase activities.

\section{Functions of 14-3-3}

14-3-3 appears to be a crucial cellular regulator via its widespread interactions with hundreds of proteins that are known to modulate an array of cellular events in multiple epithelia/endothelia (Table 2). The numerous binding partners of 14-3-3 can be categorized into proteins related to i) intracellular trafficking, ii) cell-junction dynamics, and iii) cell polarity, all of them suggesting the involvement of 14-3-3 in corresponding physiological events (Fig. 2). Such involvement is likely important for spermatogenesis because some of these 14-3-3 binding partners are implicated in germ cell movement and cell polarity during spermiogenesis and spermiation (Wong et al. 2008a).

\section{Intracellular trafficking}

As discussed above, Cdc25C protein can be docked in the cytoplasm via its binding to 14-3-3. This is one method by which 14-3-3 regulates protein localization. 14-3-3 also regulates cellular protein localization by modulating endoplasmic reticulum (ER) transportation and, thereby, the subsequent cell-surface expression of its binding partners. The membrane-trafficking mechanism involves retrograde 
transport mediated by coat protein complex I (COPI) and anterograde transport mediated by coat protein complex II (COPII). If a cargo protein has a significant forward trafficking signal, it will interact with COPII and proceed to the Golgi bodies and other intracellular destinations (Barlowe 2003, Bonifacino \& Glick 2004). However, if this cargo protein expresses an ER localization signal, it will be recognized by COPI and transported back to the ER (Ma et al. 2001).

14-3-3 has also been found to bind to a number of membrane proteins and modify their cell-surface expression levels (Mrowiec \& Schwappach 2006). The first evidence of this important function came from studies of the potassium channel protein KCNK3 (TASK-1). KCNK3 is a multimeric protein with an $\mathrm{N}$-terminal ER localization signal (i.e. COPI interacting motif) and a C-terminal 14-3-3 binding site (O'Kelly et al. 2002, Rajan et al. 2002). Truncation of the C-terminal 14-3-3 binding site of KCNK3 was shown to alter its cell-surface expression (Rajan et al. 2002), thereby modifying the $\mathrm{K}^{+}$-channel function. Moreover, cell-surface expression of TASK-1 was shown to be abolished with the mutation of serine at the -1 site at the $\mathrm{N}$-terminus (Rajan et al. 2002). In another report, the interaction between KCNK3 and 14-3-3 or COPI was found to be mutually exclusive (O'Kelly et al. 2002). All this evidence suggests that unphosphorylated KCNK3 is recognized by COPI and retained on the ER, whereas 14-3-3 binding with phosphorylated KCNK3 can prevent COPI recognition and allow its cell-surface expression (Mrowiec \& Schwappach 2006, Shikano et al. 2006). This thus provides a unique mechanism to regulate the $\mathrm{K}^{+}$-channel function by regulating the steady-state level of KCNK3 protein. Several other membrane proteins, such as the MHC II, $\mathrm{K}_{\mathrm{ATP}}$-channel subunit, and kainate receptor subunit, have been reported to be subjected to similar regulation in their cell-surface expression (Anderson et al. 1999, Yuan et al. 2003, Vivithanaporn et al. 2006). Furthermore, some evidence suggests that 14-3-3 may also regulate the interaction between COPII and its cargo (Shikano et al. 2006; Fig. 2A). Considering the numerous binding partners of 14-3-3, we suggest that 14-3-3 may function in the seminiferous epithelium during spermatogenesis by regulating protein localization.

\section{Cell-junction dynamics}

14-3-3 has been reported to be involved in regulating celljunction dynamics. One example of such regulation is its involvement in integrin-mediated cell adhesion (Fig. 2B). $\mathrm{p} 130^{\text {Cas }}$ is a docking protein that can form intracellular signaling complexes through integrin-mediated cell adhesion. In both yeast and mammalian cells, 14-3-3 has been found to be associated with $\mathrm{p} 130^{\text {Cas }}$ in a phosphoserine-dependent manner (Garcia-Guzman et al. 1999). Induced by the attachment of cells to the extracellular matrix, p130 Cas and 14-3-3 were found to be significantly co-localized upon integrin-ligand binding (Garcia-Guzman et al. 1999). Thus, 14-3-3 seems to participate in the integrin-activated signaling pathways via $\mathrm{p} 130^{\mathrm{Cas}}$ and to further regulate subsequent cell adhesion responses (Fig. 2B). In the testis, the $\alpha 6 \beta 1$-integrin/ laminin-333 adhesion complex, wherein $\alpha 6 \beta 1$-integrin residing in Sertoli cells and laminin-333 (laminin $\alpha 3 / \beta 3$ / $\gamma 3)$ restricted to elongating spermatids (Yan \& Cheng 2006), is one of the best studied adhesion complexes at the apical ES (Cheng \& Mruk 2002, Mruk et al. 2008, Vogl et al. 2008). Recent studies have shown that the anchoring junction restructuring in the seminiferous epithelium mediated by the integrin/laminin protein complex involves the activation of the integrin downstream adaptors, such as $\mathrm{p} 130^{\mathrm{Cas}}$, which recruit ERK to the apical ES to elicit junction restructuring (Siu et al. 2003, 2005). It was not known at the time how $\mathrm{p} 130^{\text {Cas }}$ can be docked at the apical ES to carry out its function. Since 14-3-3 has been shown to be abundant at

Table 2 Different classes of binding partners of 14-3-3 illustrating its potential role in regulating various cellular functions ${ }^{a}$

\section{Binding partner categories}

Polarity proteins

Cell-junction proteins

Cytoskeleton

Cytoskeleton-regulating proteins

Signaling proteins

ER localization

\section{Reported binding partners}

Par1/MARK kinase protein families (Brajenovic et al. 2004, Jin et al. 2004, Goransson et al. 2006)

Par3/Par6/aPKC complex (Brajenovic et al. 2004, Jin et al. 2004)

p120 catenin (Jin et al. 2004)

Plakophilin ( Jin et al. 2004)

AF6/afadin (Jin et al. 2004)

Actin and tubulin (Jin et al. 2004, Meek et al. 2004)

Kinesin and profilin (Jin et al. 2004, Meek et al. 2004)

Pl-3 kinase (Meek et al. 2004)

p130 Cas (Garcia-Guzman et al. 1999)

Raf kinase (Berruti 2000)

COP1 and COP2 (Shikano et al. 2006)

${ }^{a}$ This table is not intended to be exhaustive since more than a hundred binding partners for 14-3-3 have been identified in different epithelia (Jin et al. 2004 , Meek et al. 2004) based on studies using gene-profiling techniques, among others. Only selected binding partners are listed here which are known to regulate spermatogenesis based on recent studies in the field. 
the apical ES (Fig. 1), it is likely that 14-3-3 plays a major role in recruiting $\mathrm{p} 130^{\mathrm{Cas}}$ and other regulatory proteins (e.g. ERK MAP kinase) to the apical ES to facilitate its restructuring during spermatid movement in spermiogenesis and spermiation. This possibility should be carefully evaluated in future studies.

\section{Cell polarity}

Cell-polarity proteins, such as Par1, Par3, Par6, and Par5 (i.e. members of 14-3-3 in Caenorhabditis elegans), play critical roles in cell polarization. Notably, the Par3/Par6/aPKC complex is a known binding partner of 14-3-3. During cellcell contact assembly, components of the Par3/Par6/aPKC complex are brought sequentially into contact with the adhesion junction-associated molecules (Assemat et al. 2007,

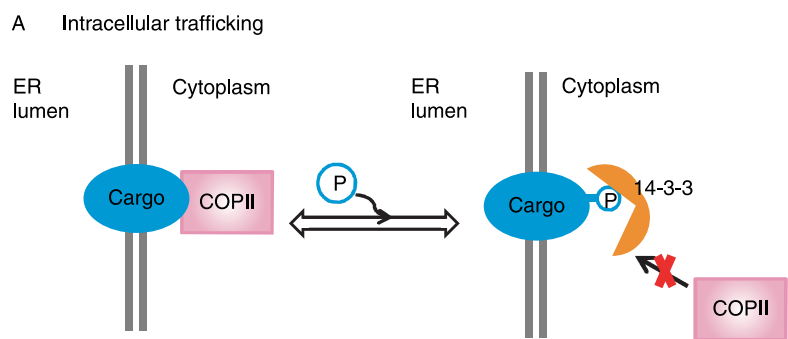

B Cell junction dynamics
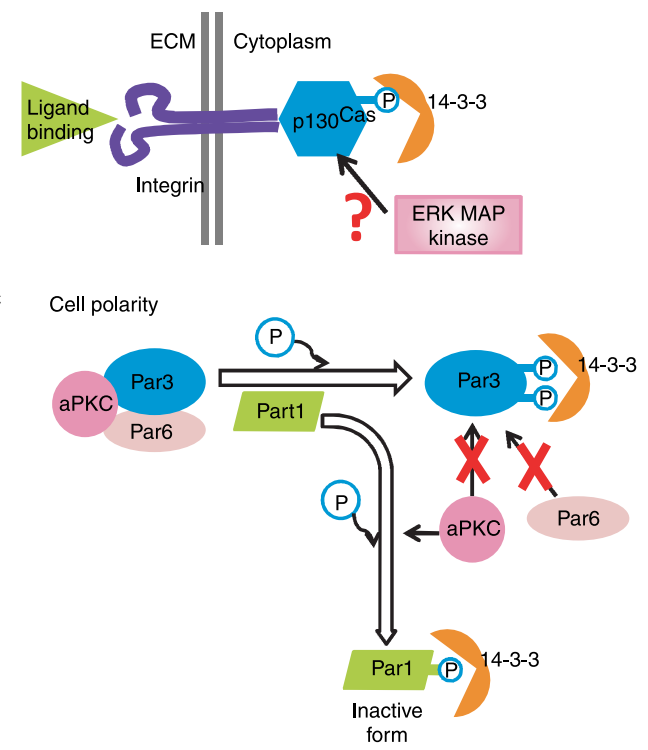

Figure 2 The three possible regulatory functions of 14-3-3 in the testis. (A) Effects on intracellular trafficking. 14-3-3 binding modulates endoplasmic reticulum (ER) transportation by masking the COPII (or COPI) binding sites on cargo proteins. (B) Effects on cell-junction dynamics. 14-3-3 participates in the integrin-activated signaling pathways via 130 Cas . (C) Effects on cell polarity. Par1 catalyzes the phosphorylation of Par3, leading to 14-3-3 binding that inhibits the formation of Par3/Par6/aPKC complex. In turn, Par1 can be phosphorylated by aPKC (atypical protein kinase $\mathrm{C}$ ) and bound with 14-3-3 as inactive form.
Ebnet et al. 2008; Fig. 2C). In the testis, Par3, Par6, and aPKC have been identified by corresponding specific antibodies in Sertoli and germ cells in the seminiferous epithelium of adult rats (Wong et al. 2008a). The consequent Par3/Par6/aPKC complex has been shown to serve as a crucial polarity complex at the apical ES to confer spermatid orientation, so that the heads of the developing spermatids in the seminiferous epithelium are properly oriented towards the basement membrane (Wong et al. 2008a). More important, it was shown that this Par3/Par6-based polarity complex is also working in concert with proteins at the apical ES (e.g. JAM-C) and proteins at the BTB (e.g. JAM-A and $N$-cadherin); as a result, the cellular events of spermiation and BTB restructuring that occur simultaneously at the opposite ends of the Sertoli cell epithelium at stage VIII of the seminiferous epithelial cycle can be precisely coordinated (Wong et al. 2008a). While the precise downstream mechanism(s) that coordinates these two concurrent cellular events involving the Par-based polarity complex remains to be elucidated, it is likely that 14-3-3 and two non-receptor protein tyrosine kinases $-\mathrm{c}-\mathrm{Src}$ and FAK and their corresponding activated forms $\mathrm{p}-\mathrm{cSrc}$ and $\mathrm{p}-\mathrm{FAK}-$ are involved. For instance, it is known that c-Src and FAK are components of the BTB (Siu et al. 2003, Lee \& Cheng 2005), whereas their activated $\mathrm{p}-\mathrm{cSrc}$ and $\mathrm{p}$-FAK forms are found mostly at the apical ES (Siu et al. 2003, Wong et al. 2005, Beardsley et al. 2006). It is possible that 14-3-3 serves as the docking platform for protein kinases (e.g. c-Src and FAK) and their activated forms to mediate the junction restructuring events that facilitate spermiation at the apical ES and the transit of preleptotene spermatocytes at the BTB at stage VIII of the cycle. This concept should be vigorously tested in future experiments.

Par3 can be phosphorylated by Par1 on the Ser-151 and Ser-1085 residues. These two phosphorylated Ser residues are the putative binding sites for Par5/14-3-3 (Benton \& Johnston 2003, Hurd et al. 2003), allowing these proteins to form a protein complex (Fig. 2C). The binding of 14-3-3 with the Ser-151 residue blocks the oligomerization and functioning of Par3, and the masking of the Ser-1085 residue inhibits the binding of aPKC (Nagai-Tamai et al. 2002, Benton \& Johnston 2003, Brajenovic et al. 2004; Fig. 2C). Consequently, if the cell membrane domain has active Par1 proteins associated, the phosphorylated Par3 will be incapable of forming a functional Par3/Par6/aPKC complex at the cell membrane (Ebnet et al. 2008; Fig. 2C), leading to changes in polarity.

Interestingly, Par1 itself can also be phosphorylated by aPKC at Thr-595 and bind to 14-3-3. The binding inhibits the kinase activity of Par1 and releases it from cell membrane (Hurov et al. 2004, Suzuki et al. 2004). Using this mechanism, the functional Par3/Par6/aPKC complex can be localized at the desired cellular site (Fig. 2C). Another study has shown several other phosphorylation sites on Par1 to be important for 14-3-3 binding, and the simultaneous mutation of those phosphorylation sites restricted Par1 at the plasma membrane 


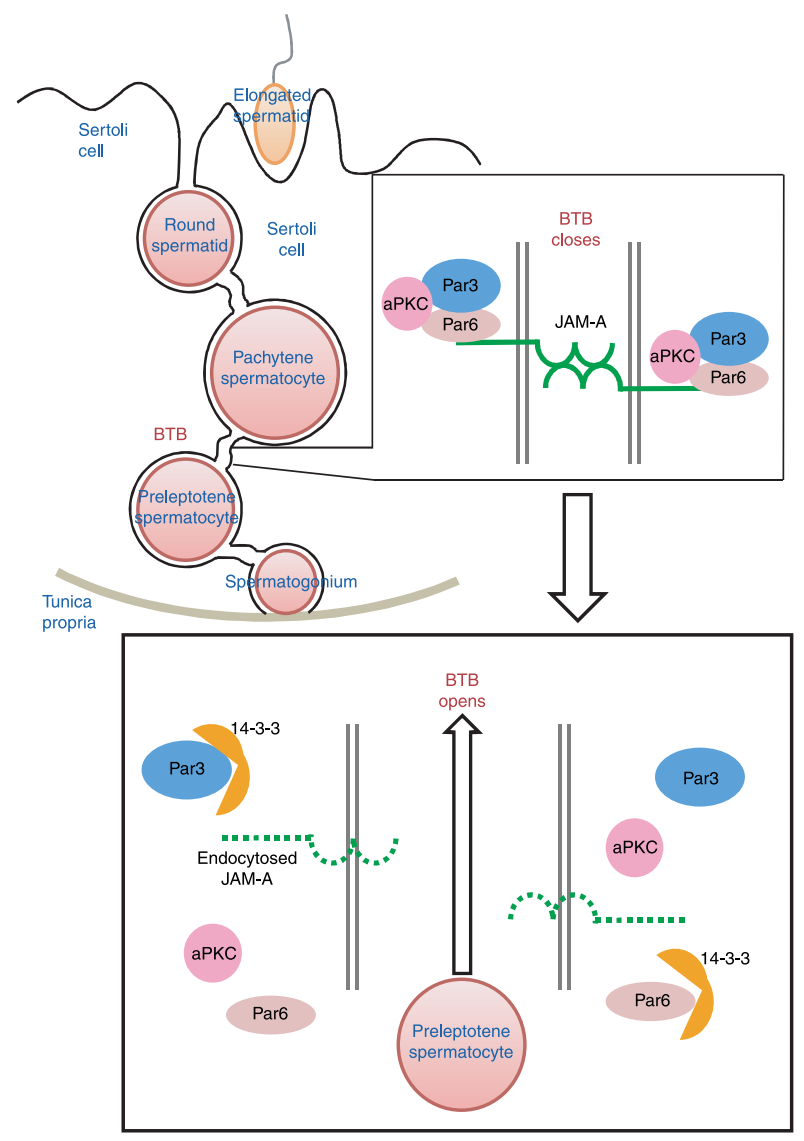

Figure 3 A hypothetical model illustrating the possible regulatory role of 14-3-3 during BTB restructuring at stage VIII of the seminiferous epithelial cycle of spermatogenesis. The direct contact between the Par3/Par6/aPKC complex and JAM-A stabilizes the BTB, conferring an intact barrier. At stage VIII of the epithelial cycle, the degradation or endocytosis of JAM-A (dashed line) induces the opening of BTB. This diminishing level of JAM-A at the BTB may be contributed by the disassembly of the Par3/Par6/aPKC protein complex, because of the masking of Par3 and/or Par6 by 14-3-3 protein.

(Goransson et al. 2006). These results suggest that complex interactions exist among polarity proteins, facilitating the formation and/or dissolution of the polarity protein complex during the epithelial cycle of spermatogenesis, and that 14-3-3 is a crucial regulator mediating protein-protein interactions. For instance, a study has reported that Par6, Pals1, and JAM-C interact at the apical ES in normal rat testes (Wong et al. 2008a). However, at the time of germ cell depletion from the epithelium induced by adjudin, Par6, Src, and Pals1 formed a tighter protein complex, which, in turn, weakened the association of Pals1 and Par6 with JAM-C, destabilizing the JAM-C-based adhesion, and leading to germ cell loss from the epithelium (Wong et al. 2008a). It is plausible that 14-3-3 plays a crucial role in the mediation of proteinprotein interactions at the apical ES involving Pals1, Par6, Src, and JAM-C.
In addition to its likely involvement in the apical ES restructuring, 14-3-3 was also implicated in the regulation of BTB restructuring during spermatogenesis (Wong et al. 2008a; Fig. 3). For instance, it has been shown that Par3, Par6, aPKC and JAM-A interact at the BTB to maintain cell adhesion at the site (Wong et al. 2008a). At stage VIII of the epithelial cycle, when primary preleptotene spermatocytes are in transit at the BTB, Par3/Par6/aPKC complexes disassemble, which thus destabilizes JAM-A-based cell adhesion, facilitating the transit of primary spermatocytes at the BTB (Fig. 3). It is likely that $14-3-3$ plays a role at the $\mathrm{BTB}$ in mediating protein-protein interactions involving Par3, Par6, aPKC, and/or JAM-A (Fig. 3).

\section{Regulation}

The regulation of 14-3-3 activity can occur either on the binding partners of 14-3-3 or on the 14-3-3 protein per se, both forms of which are carried out through changes in the phosphorylation status of specific amino acid residues.

Two important regulators involved in 14-3-3 binding are the protein phosphatases PP1 and PP2, which dephosphorylate the desired Ser or Tyr residue(s) at the 14-3-3 binding sites. In the binding between phosphorylated Par3 and 14-3-3 discussed above, PP1 plays an important regulatory role by dephosphorylating multiple sites on Par3, thus controls the binding of 14-3-3 and aPKC to Par3 (Traweger et al. 2008). A similar regulatory pathway exists for $\mathrm{Cdc} 25 \mathrm{C}$, which can be released from 14-3-3 by PP1 and subsequently translocated to the nucleus (Margolis et al. 2003).

The activity of 14-3-3 proteins can also be regulated by phosphorylation. Several protein kinases, such as protein kinase $\mathrm{B}$, protein kinase $\mathrm{C}$, and casein kinase 1 , have been shown to be capable of phosphorylating 14-3-3. Moreover, these proteins have been positively identified in the testis and are known to be expressed by Sertoli and/or germ cells (Lee \& Cheng 2005, Siu et al. 2005). For instance, since p-PKB$\mathrm{Thr}^{308}$ and $\mathrm{p}-\mathrm{PKB}-\mathrm{Ser}^{473}$ were both shown to be induced during anchoring junction disruption at the Sertoli cell-germ cell interface, the phosphorylation of 14-3-3 is likely to occur at the apical ES. It has been reported that 14-3-3 phosphorylation always results in decreased target binding

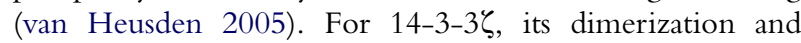
consequent activity can also be regulated by phosphorylation, in which the phosphorylated Ser58 disrupts the dimeric conformation (Woodcock et al. 2003). In short, these findings have demonstrated that the effects of $14-3-3$ on proteinprotein interactions at the apical ES and BTB in the seminiferous epithelium are regulated by its interactions with many of the recently reported adaptors (e.g. p130Cas), protein kinases (e.g. $\mathrm{PKB}$ and $\mathrm{Src}$ ), polarity proteins (e.g. Par6 and Pals1), and integral membrane proteins (e.g. JAM-A, JAM-C, and integrins). 


\section{Concluding remarks and future perspectives}

Herein, we summarize and discuss some of the latest findings regarding 14-3-3 (also known as Par5) in the epithelium including the seminiferous epithelium. Earlier studies have shown that it is a component of the polarity protein complex. However, in light of the great number of protein-protein interactions that it is involved in, 14-3-3, along with components of the polarity protein complex, which clearly have physiological functions other than conferring cell polarity. For instance, recent findings have illustrated that Par6 and Par3, the close 'cousins' of 14-3-3, are involved in spermatid release/adhesion as well as BTB dynamics, possibly coordinating the cellular events of spermiation and BTB restructuring, which occur at the opposite ends of the Sertoli cell epithelium in the testis at stage VIII of the epithelial cycle (Wong et al. 2008a). Since 14-3-3 interacts with Par3 and Par6 in the testis. This 14-3-3-based polarity complex is likely to serve as a crucial regulatory platform in the seminiferous epithelium to affect and/or coordinate many cellular events during spermatogenesis. We suggest that future studies should be directed to delineate the role of 14-3-3 in post-meiotic germ cell development, in particular spermiogenesis, since there is considerable turnover of proteins in the developing spermatids as well as at the Sertoli cell-spermatid interface.

\section{Declaration of interest}

The authors herein declare that there is no conflict of interest that could be perceived as prejudicing the impartiality of the research/work reported.

\section{Funding}

Studies from the authors' laboratory reported in this review were supported in part by grants from the National Institutes of Health (NICHD, RO1 HD056034; RO3 HD051512; and U54 HD029990 Project 5 all to C Y C). W M L was supported by a grant from Hong Kong Research Grants Council, HKU7693/07M.

\section{References}

Aitken A 2006 14-3-3 Proteins: a historic overview. Seminars in Cancer Biology 16 162-172.

Anderson H, Bergstralh D, Kawamura T, Blauvelt A \& Roche P 1999 Phosphorylation of the invariant chain by protein kinase $\mathrm{C}$ regulates $\mathrm{MHC}$ Class II trafficking to antigen-processing compartments. Journal of Immunology 163 5435-5443.

Assemat E, Bazellieres E, Pallesi-Pocachard E, Le Bivic A \& Massey-Harroche D 2007 Polarity complex proteins. Biochimica et Biophysica Acta 1778 614-630.

Athwal G, Huber J \& Huber S 1998 Phosphorylated nitrate reductase and 14-3-3 proteins site of interaction, effects of ions, and evidence for an AMP-binding site on 14-3-3 proteins. Plant Physiology 118 1041-1048.

Barlowe C 2003 Signals for COPII-dependent export from the ER: what's the ticket out? Trends in Cell Biology 13 295-300.
Beardsley A, Robertson DM \& O’Donnell P 2006 A complex containing $\alpha 6 \beta 1$-integrin and phosphorylated focal adhesion kinase between Sertoli cells and elongated spermatids during spermatid release from the seminiferous epithelium. Journal of Endocrinology 190 759-770.

Benton R \& Johnston D 2003 A conserved oligomerization domain in Drosophila Bazooka/PAR-3 is important for apical localization and epithelial polarity. Current Biology 13 1330-1334.

Benzinger A, Muster N, Koch H, Yates J \& Hermeking H 2005 Targeted proteomic analysis of 14-3-3 sigma, a p53 effector commonly silenced in cancer. Molecular and Cellular Proteomics 4 785-795.

Berruti G 2000 A novel Rap1/B-Raf/14-3-3 theta protein complex is formed in vivo during the morphogenetic differentiation of postmeiotic male germ cells. Experimental Cell Research 257 172-179.

Bonifacino J \& Glick B 2004 The mechanisms of vesicle budding and fusion. Cell 116 153-166.

Boston PF, Jackson P, Kynoch PA \& Thompson RJ 1982 Human 14-3-3 protein: radioimmunoassay, tissue distribution, and cerebrospinal fluid levels in patients with neurological disorders. Journal of Neurochemistry $\mathbf{3 8}$ 1466-1474.

Brajenovic M, Joberty G, Kuester B, Bouwmeester T \& Drewes G 2004 Comprehensive proteomic analysis of human Par protein complexes reveals an interconnected protein network. Journal of Biological Chemistry 279 12804-12811.

Bridges D \& Moorhead GB 2004 14-3-3 Proteins: a number of functions for a numbered protein. Science's STKE 242 re10.

Bridges D \& Moorhead GB 2005 Review update: 14-3-3 proteins: a number of functions for a numbered protein. Science's STKE 296 re10.

Chapin RE, Wine RN, Harris MW, Borchers CH \& Haseman JK 2001 Structure and control of a cell-cell adhesion complex associated with spermiation in rat seminiferous epithelium. Journal of Andrology 22 1030-1052.

Chaudhri M, Scarabel M \& Aitken A 2003 Mammalian and yeast 14-3-3 isoforms form distinct patterns of dimers in vivo. Biochemical and Biophysical Research Communications 300 679-685

Cheng CY \& Mruk DD 2002 Cell junction dynamics in the testis: Sertoligerm cell interactions and male contraceptive development. Physiological Reviews 82 825-874.

Dougherty MK \& Morrison DK 2004 Unlocking the code of 14-3-3. Journal of Cell Science 117 1875-1884.

Ebnet K, Iden S, Gerke V \& Suzuki A 2008 Regulation of epithelial and endothelial junctions by PAR proteins. Frontiers in Bioscience 13 6520-6536.

Fu H, Subramanian RR \& Masters SC 2000 14-3-3 Proteins: structure, function, and regulation. Annual Review of Pharmacology and Toxicology 40 617-647.

Ganguly S, Weller J, Ho A, Chemineau P, Malpaux B \& Klein D 2005 Melatonin synthesis: 14-3-3-dependent activation and inhibition of arylalkylamine $N$-acetyltransferase mediated by phosphoserine- 205 . PNAS 102 1222-1227.

Garcia-Guzman M, Dolfi F, Russello M \& Vuori K 1999 Cell adhesion regulates the interaction between the docking protein $\mathrm{p} 130 \mathrm{Cas}$ and the 14-3-3 proteins. Journal of Biological Chemistry 274 5762-5768.

Goransson O, Deak M, Wullschleger S, Morrice NA, Prescott AR \& Alessi DR 2006 Regulation of the polarity kinases PAR-1/MARK by 14-3-3 interaction and phosphorylation. Journal of Cell Science 1194059.

van Heusden G 2005 14-3-3 Proteins: regulators of numerous eukaryotic proteins. IUBMB Life 57 623-629.

Hess R \& de Franca LR 2008 Spermatogenesis and cycle of the seminiferous epithelium. In Molecular Mechanisms in Spermatogenesis, pp 1-15. Ed. CY Cheng. Austin, Texas: Landes BioScience and Springer Science + Business Media LLC.

Hurd T, Fan S, Liu C, Kweon H, Hakansson K \& Margolis B 2003 Phosphorylation-dependent binding of 14-3-3 to the polarity protein Par3 regulates cell polarity in mammalian epithelia. Current Biology 13 2082-2090.

Hurov J, Watkins J \& Piwnica-Worms H 2004 Atypical PKC phosphorylates PAR-1 kinases to regulate localization and activity. Current Biology 14 736-741. 
Jin J, Smith FD, Stark C, Wells CD, Fawcett JP, Kulkarni S, Metalnikov P, O’Donnell P, Taylor P \& Taylor L 2004 Proteomic, functional, and domain-based analysis of in vivo 14-3-3 binding proteins involved in cytoskeletal regulation and cellular organization. Current Biology 14 1436-1450.

Jones DH, Ley S \& Aitken A 1995 Isoforms of 14-3-3 protein can form homo- and heterodimers in vivo and in vitro: implications for function as adapter proteins. FEBS Letters 368 55-58.

Kjarland E, Keen TJ \& Kleppe R 2006 Does isoform diversity explain functional differences in the 14-3-3 protein family? Current Pharmaceutical Biotechnology 7 217-223.

Kumagai A \& Dunphy W 1999 Binding of 14-3-3 proteins and nuclear export control the intracellular localization of the mitotic inducer Cdc25. Genes and Development 13 1067-1072.

Lee NPY \& Cheng CY 2004 Adaptors, junction dynamics, and spermatogenesis. Biology of Reproduction 71 392-404.

Lee NPY \& Cheng CY 2005 Protein kinases and adherens junction dynamics in the seminiferous epithelium of the rat testis. Journal of Cellular Physiology 202 344-360.

Liu D, Bienkowska J, Petosa C, Collier R, Fu H \& Liddington R 1995 Crystal structure of the zeta isoform of the 14-3-3 protein. Nature 376 191-194.

Ma D, Zerangue N, Lin Y, Collins A, Yu M, Jan Y \& Jan L 2001 Role of ER export signals in controlling surface potassium channel numbers. Science $\mathbf{2 9 1}$ 316-319.

Mackintosh C 2004 Dynamic interactions between 14-3-3 proteins and phosphoproteins regulate diverse cellular processes. Biochemical Journal $\mathbf{3 8 1}$ 329-342.

Margolis S, Walsh S, Weiser D, Yoshida M, Shenolikar S \& Kornbluth S 2003 PP1 control of M phase entry exerted through 14-3-3-regulated Cdc25 dephosphorylation. EMBO Journal 22 5734-5745.

Masters S, Pederson K, Zhang L, Barbieri J \& Fu H 1999 Interaction of 14-3-3 with a nonphosphorylated protein ligand, exoenzyme S of Pseudomonas aeruginosa. Biochemistry 38 5216-5221.

McKinsey T, Zhang C \& Olson E 2001 Identification of a signal-responsive nuclear export sequence in Class II histone deacetylases. Molecular and Cellular Biology 21 6312-6321.

Meek SEM, Lane WS \& Piwnica-Worms H 2004 Comprehensive proteomic analysis of interphase and mitotic 14-3-3-binding proteins. Journal of Biological Chemistry 27932046.

Moore BW \& Perez VJ 1967 Specific acidic proteins of the nervous system. In Physiological and Biochemical Aspects of Nervous Integration, pp 343-359. Ed. FD Carlson. Englewood Cliffs, NJ: Prentice Hall.

Moorhead G, Douglas P, Morrice N, Scarabel M, Aitken A \& MacKintosh C 1996 Phosphorylated nitrate reductase from spinach leaves is inhibited by 14-3-3 proteins and activated by fusicoccin. Current Biology 6 1104-1113.

Mrowiec T \& Schwappach B 2006 14-3-3 Proteins in membrane protein transport. Biological Chemistry 387 1227-1236.

Mruk DD \& Cheng CY 2004 Sertoli-Sertoli and Sertoli-germ cell interactions and their significance in germ cell movement in the seminiferous epithelium during spermatogenesis. Endocrine Reviews 25 747-806.

Mruk DD, Silvestrini B \& Cheng CY 2008 Anchoring junctions are drug targets: role in contraceptive development. Pharmacological Reviews $\mathbf{6 0}$ 146-180.

Nagai-Tamai Y, Mizuno K, Hirose T, Suzuki A \& Ohno S 2002 Regulated protein-protein interaction between aPKC and PAR-3 plays an essential role in the polarization of epithelial cells. Genes to Cells 7 1161-1171.

Niemantsverdriet M, Wagner K, Visser M \& Backendorf C 2008 Cellular functions of 14-3-3zeta in apoptosis and cell adhesion emphasize its oncogenic character. Oncogene 27 1315-1319.

Obsil T, Ghirlando R, Klein D, Ganguly S \& Dyda F 2001 Crystal structure of the 14-3-3zeta: serotonin $N$-acetyltransferase complex: a role for scaffolding in enzyme regulation. Cell 105 257-267.

O'Kelly I, Butler M, Zilberberg N \& Goldstein S 2002 Forward transport 14-3-3 binding overcomes retention in endoplasmic reticulum by dibasic signals. Cell $111577-588$.
Peng C, Graves P, Thoma R, Wu Z, Shaw A \& Piwnica-Worms H 1997 Mitotic and G2 checkpoint control: regulation of 14-3-3 protein binding by phosphorylation of Cdc25C on Serine-216. Science 2771501.

Perego L \& Berruti G 1997 Molecular cloning and tissue-specific expression of the mouse homologue of the rat brain 14-3-3 theta protein: characterization of its cellular and developmental pattern of expression in the male germ line. Molecular Reproduction and Development 47 370-379.

Petosa C, Masters S, Bankston L, Pohl J, Wang B, Fu H \& Liddington R 1998 14-3-3zeta binds a phosphorylated Raf peptide and an unphosphorylated peptide via its conserved amphipathic groove. Journal of Biological Chemistry 273 16305-16310.

Rajan S, Preisig-Muller R, Wischmeyer E, Nehring R, Hanley P, Renigunta V, Musset B, Schlichthorl G, Derst C \& Karschin A 2002 Interaction with 14-3-3 proteins promotes functional expression of the potassium channels TASK-1 and TASK-3. Journal of Physiology 545 13-26.

Rittinger K, Budman J, Xu J, Volinia S, Cantley L, Smerdon S, Gamblin S \& Yaffe M 1999 Structural analysis of 14-3-3 phosphopeptide complexes identifies a dual role for the nuclear export signal of 14-3-3 in ligand binding. Molecular Cell 4 153-166.

Rosenquist M, Sehnke P, Ferl RJ, Sommarin M \& Larsson C 2000 Evolution of the 14-3-3 protein family: does the large number of isoforms in multicellular organisms reflect functional specificity? Journal of Molecular Evolution 51 446-458.

Rosenquist M, Alsterfjord M, Larsson C \& Sommarin M 2001 Data mining the Arabidopsis genome reveals fifteen 14-3-3 genes. Expression is demonstrated for two out of five novel genes. Plant Physiology 127 142-149.

Shikano S, Coblitz B, Wu M \& Li M 2006 14-3-3 Proteins: regulation of endoplasmic reticulum localization and surface expression of membrane proteins. Trends in Cell Biology 16 370-375.

Siu MKY, Mruk DD, Lee WM \& Cheng CY 2003 Adhering junction dynamics in the testis are regulated by an interplay of $\beta 1$-integrin and focal adhesion complex-associated proteins. Endocrinology 144 2141-2163.

Siu MKY, Wong CH, Lee WM \& Cheng CY 2005 Sertoli-germ cell anchoring junciton dynamics in the testis are regulated by an interplay of lipid and protein kinases. Journal of Biological Chemistry 280 25029-25047.

Suzuki A, Hirata M, Kamimura K, Maniwa R, Yamanaka T, Mizuno K, Kishikawa M, Hirose H, Amano Y \& Izumi N 2004 aPKC acts upstream of PAR- $1 \mathrm{~b}$ in both the establishment and maintenance of mammalian epithelial polarity. Current Biology 14 1425-1435.

Traweger A, Wiggin G, Taylor L, Tate SA, Metalnikov P \& Pawson T 2008 Protein phosphatase 1 regulates the phosphorylation state of the polarity scaffold Par-3. PNAS 105 10402-10407.

Vivithanaporn P, Yan S \& Swanson G 2006 Intracellular trafficking of KA2 kainate receptors mediated by interactions with coatomer protein complex I (COPI) and 14-3-3 chaperone systems. Journal of Biological Chemistry 281 15475-15484.

Vogl AW, Vaid KS \& Guttman JA 2008 The Sertoli cell cytoskeleton. In Molecular Mechanisms in Spermatogenesis, pp 186-211. Ed. CY Cheng. Austin, TX: Landes Bioscience/Springer Bioscience.

Wang WS \& Shakes DC 1996 Molecular evolution of the 14-3-3 protein family. Journal of Molecular Evolution 43 384-398.

Wine RN \& Chapin RE 1999 Adhesion and signaling proteins spatiotemporally associated with spermiation. Journal of Andrology 20 198-213.

Wong CH, Xia W, Lee NPY, Mruk DD, Lee WM \& Cheng CY 2005 Regulation of ectoplasmic specialization dynamics in the seminiferous epithelium by focal adhesion-associated proteins in testosterone-suppressed rat testes. Endocrinology 146 1192-1204.

Wong EW, Mruk DD, Lee WM \& Cheng CY 2008a Par3/Par6 polarity complex coordinates apical ectoplasmic specialization and blood-testis barrier restructuring during spermatogenesis. PNAS 105 9657-9662.

Wong EWP, Mruk DD \& Cheng CY 2008b Biology and regulation of ectoplasmic specialization, an atypical adherens junction type, in the testis. Biochimica et Biophysica Acta 1778 692-708.

Woodcock JM, Murphy J, Stomski FC, Berndt MC \& Lopez AF 2003 The dimeric versus monomeric status of 14-3-3 zeta is controlled by phosphorylation of Ser58 at the dimer Interface ${ }^{\star}$. Journal of Biological Chemistry 278 36323-36327. 
Xiao B, Smerdon SJ, Jones DH, Dodson GG, Soneji Y, Aitken A \& Gamblin SJ 1995 Structure of a 14-3-3 protein and implications for coordination of multiple signalling pathways. Nature 376 188-191.

Yaffe M 2002 How do 14-3-3 proteins work? - gatekeeper phosphorylation and the molecular anvil hypothesis FEBS Letters 513 53-57.

Yaffe M, Rittinger K, Volinia S, Caron P, Aitken A, Leffers H, Gamblin S, Smerdon S \& Cantley L 1997 The structural basis for 14-3-3: phosphopeptide binding specificity. Cell 91 961-971.

Yan HHN \& Cheng CY 2006 Laminin $\alpha 3$ forms a complex with $\beta 3$ and $\gamma 3$ chains that serves as the ligand for $\alpha 6 \beta 1$-integrin at the apical ectoplasmic specialization in adult rat testes. Journal of Biological Chemistry 281 17286-17303.
Yuan H, Michelsen K \& Schwappach B 2003 14-3-3 dimers probe the assembly status of multimeric membrane proteins. Current Biology $\mathbf{1 3}$ 638-646.

Zhang J, Mruk DD \& Cheng CY 2005 Myotubularin phosphoinositide phosphatases, protein phosphatases, and kinases: their roles in junction dynamics and spermatogenesis. Journal of Cellular Physiology 204 470-483.

Received in final form 2 April 2009

Accepted 14 April 2009

Made available online as an Accepted Preprint 14 April 2009 